\title{
Desarrollo de la regeneración a lo largo del ciclo del manejo forestal de un bosque de Nothofagus pumilio: 1. Incidencia de la cobertura y el aprovechamiento o cosecha
}

\author{
Regeneration of Nothofagus pumilio throughout the forest management cycle: \\ 1. Crown closure and harvesting incidence \\ GUILLERMO MARTINEZ-PASTUR ${ }^{1}$, PABLO LUIS PERI ${ }^{2,3}$, \\ MARIA CECILIA FERNANDEZ ${ }^{1,3}$, GABRIELA STAFFIERI ${ }^{4}$ \\ ${ }^{1}$ Centro Austral de Investigaciones Científicas (CONICET), cc 92 (9410) Ushuaia, \\ Tierra del Fuego (Argentina). ${ }^{2}$ Universidad Nacional de la Patagonia Austral. \\ ${ }^{3}$ Instituto Nacional de Tecnología Agropecuaria. ${ }^{4}$ Universidad Nacional de La Plata.
}

\author{
SUMMARY
}

\begin{abstract}
Lenga is the forest species with the highest economical impact in Patagonia. Within the forest, an important population of saplings and seedlings whose survival depends on the quantity of light that the parent tree canopy allows to enter, can be found. Their regeneration can be affected by the harvesting. The objective of this work was to analyse the influence of the crown closure and harvesting on the installation and evolution of natural regeneration in a Nothofagus pumilio forest managed by a shelterwood system in Tierra del Fuego. The amount of seedlings and saplings in the studied forests was abundant for forest management purposes. The crown closure significantly affected the installation and growth of seedlings and saplings. On the other hand, it is possible to find a bank of regeneration in the understory, that would be enough to regenerate the forest if a shelterwood system is carried out. It is very important to consider the amount of saplings and seedlings in the decisions of a forest management plan because harvesting activities affect the installed saplings and seedlings.
\end{abstract}

Key words: regeneration, Nothofagus pumilio, shelterwood cut system, crown closure, harvesting.

\section{RESUMEN}

La Lenga es la especie forestal de mayor impacto económico en la Patagonia. Dentro del bosque es posible encontrar una población importante de renovales, cuya supervivencia depende de la cantidad de luz que el dosel arbóreo deja ingresar. Esta regeneración preinstalada puede ser afectada por las tareas de extracción de madera. El objetivo de este trabajo fue analizar la incidencia de la cobertura del dosel arbóreo y de las actividades de aprovechamiento o cosecha forestal sobre la instalación y supervivencia de la regeneración natural de un bosque de Nothofagus pumilio sometido a una corta de protección en Tierra del Fuego. La regeneración de los bosques estudiados es abundante y suficiente. La cobertura del bosque afecta significativamente la instalación y el crecimiento de las plántulas. Por otra parte, es posible encontrar un banco de plántulas, que es suficiente para regenerar el bosque si se realiza una corta de protección, debiéndose tener en cuenta en la toma de decisiones de un plan de manejo forestal, ya que las tareas de aprovechamiento o cosecha afectan significativamente a los renovales instalados.

Palabras claves: regeneración, Nothofagus pumilio, corta de protección, cobertura, aprovechamiento, cosecha. 


\section{INTRODUCCION}

La lenga (Nothofagus pumilio) es la especie forestal de mayor impacto económico dentro de la industria maderera de la Patagonia. Presenta un amplio rango de distribución natural que va desde $\operatorname{los} 35^{\circ} 35^{\prime}$ hasta $\operatorname{los} 55^{\circ} 31^{\prime} \mathrm{LS}$, siendo reconocida por la calidad de su madera y la gran aceptación en el mercado internacional (Rechene y Gonda 1992, Araya 1996). La lenga es una especie heliófila de tolerancia media (Rusch 1992) que posee ciclos de regeneración por bosquetes, por lo que es común encontrar abundante regeneración donde se ha producido algún desmoronamiento que permita la entrada de luz (Rebertus y Veblen 1993). La corta de reproducción que emula dicha dinámica es el sistema de cortas de protección (Schmidt y Urzúa 1982), que abre el dosel lo suficiente como para permitir el óptimo desarrollo de los renovales. Dentro de un bosque virgen podemos encontrar un banco de renovales en el sotobosque, que se va renovando a lo largo de los años y que será el primero en reaccionar al realizar una intervención silvícola de corta y regeneración. Las tareas de aprovechamiento forestal pueden afectar significativamente esta regeneración preinstalada, ya que al procesar y arrastrar los rollizos se producen notables alteraciones y daños en las plántulas, el suelo y el sotobosque (Schmidt et al. 1992, Vatasan 1983).

La regeneración que se encuentra en el sotobosque se cuantifica en los inventarios forestales, para así caracterizar la aptitud de respuesta de recuperación del rodal ante un disturbio generalizado, como lo es una corta de protección. Sin embargo, las pérdidas que se producen en el banco de renovales del bosque debidas a las tareas de aprovechamiento pocas veces son tenidas en cuenta, siendo de suma importancia para una correcta toma de decisiones dentro de un plan de manejo forestal.

El objetivo de este trabajo fue analizar la incidencia de la cobertura del dosel arbóreo y de las actividades de los aprovechamientos forestales sobre la instalación y supervivencia en los primeros años de la regeneración natural de un bosque de Nothofagus pumilio sometido a una corta de protección en Tierra del Fuego (Argentina).

\section{MATERIAL Y METODOS}

Area bajo estudio. Se trabajó en un bosque puro de $N$. pumilio de la estancia San Justo (Tierra del
Fuego) (54 $\left.06^{\prime} \mathrm{LS}, 68^{\circ} 37^{\prime} \mathrm{LO}\right)$, donde actualmente se encuentran las instalaciones del aserradero "Los Castores". Esta empresa realiza el aprovechamiento de los bosques de acuerdo a la reglamentación de la ley forestal $\mathrm{N}^{\circ} 145$ de la provincia de Tierra del Fuego, utilizando como método de reproducción al monte alto bajo cubierta protectora (corta de protección).

Selección de los rodales. Se seleccionaron tres rodales que representan momentos característicos del ciclo de manejo de la lenga, siguiendo un método de corta de protección según lo propuesto por Schmidt y Urzúa (1982). Como tiempo cero se seleccionó un rodal de un bosque virgen (BV). El segundo rodal se seleccionó en un bosque aprovechado (año forestal 1993-1994) (BA) mediante el mismo sistema silvícola actual. El tercer rodal se seleccionó en un fustal (F), un área donde se practicó una tala rasa (hace 80-85 años) que originó una estructura similar a la que se esperaría para los otros aprovechamientos, con la salvedad de que no se realizaron cortas intermedias para conducir a la masa forestal. Después de caracterizar la regeneración del bosque virgen (BV) se realizó la corta del bosque y el arrastre de los rollizos, caracterizando los efectos sobre la regeneración después del aprovechamiento forestal (BRA) para evaluar la magnitud de su incidencia. La estructura de cada rodal se caracterizó mediante el área basal (AB), el número de árboles (ARB), el diámetro cuadrático medio (DCM), el volumen total con corteza (VTCC) mediante las funciones propuestas por Peri et al. (1997) y la clase de calidad de sitio (CS) de acuerdo a la clasificación propuesta por Martínez Pastur et al. (1997), expresada como la altura que poseía el rodal a una edad base de 60 años $\left(\mathrm{IS}_{60}\right)$.

Diseño del muestreo. En cada situación se evaluó la regeneración existente mediante el muestreo sistemático de una hectárea para cada rodal. Se definieron cuatro transectas de 100 metros, distanciadas 25 entre sí. En cada transecta se realizaron seis bloques de cuatro parcelas de $0.25 \mathrm{~m}^{2}$. Cada parcela fue tomada a $2.5 \mathrm{~m}$ del centro del bloque (paralela y perpendicularmente a la dirección de las transectas). En cada parcela se determinó el número de plántulas (NP), las estructuras de edad (mediante el conteo de mucrones) (E) y altura (A). Por otra parte, se estimó el grado de cobertura de residuos que cubrían el suelo (Res) mediante una 
grilla de puntos y el porcentaje de cobertura de copas (Cob) (mediciones hechas en verano) por medio de un medidor de copas ("spherical densiometer") (Lemmon 1957).

Análisis estadístico. Con los datos del muestreo se realizó un análisis de varianza (considerando como factores principales al tipo de rodal, la historia de los aprovechamientos o la cobertura) mediante la Prueba de F, utilizando el test de Tukey para separar las medias. La probabilidad usada en todos los test fue de $\mathrm{p}<0.05$.

\section{RESULTADOS Y DISCUSION}

Caracterización de la estructura de los rodales estudiados. El bosque virgen (BV) posee la estructura de un bosque maduro irregular de clase de sitio III $\left(\right.$ IS $\left._{60}=13.1-16.5 \mathrm{~m}\right)$ con dos estratos bien diferenciados y abundante regeneración por bosquetes (cuadro 1), mientras que el fustal (F) es un bosque coetáneo de clase de sitio I ( IS $_{60}=19.8-23.2 \mathrm{~m}$ ), con un estrato y sin aperturas importantes en el dosel. El valor de densidad promedio en el bosque virgen fue de $92.86 \%$ de acuerdo al índice de densidad de Reineke (IDR) propuesto por Fernández et al. (1997), lo que indica que se trata de un rodal de densidad normal o completa. Luego de realizar el aprovechamiento (BRA) la densidad del rodal disminuyó considerablemente hasta un IDR de $39.9 \%$. Al cabo de seis años el IDR se ubicó en un valor de $19.46 \%$ (BA), debido a los volteos de viento que usualmente se producen en los primeros años posteriores a la corta de protección. El bosque virgen (BV) se puede clasificar como un bosque en envejecimiento $(53.6 \%$ de los árboles se encontraban en dicha fase), con un bajo porcentaje de individuos en fase de desmoronamiento (6.3\%) que en su mayoría fueron dejados como dosel protector luego de la corta (BRA, BA). E1 fustal (F) es un bosque en crecimiento óptimo final, ya que salvo un escaso estrato remanente (10 árboles por hectárea) la totalidad de los individuos se encuentran en dicha fase.

Los valores presentados en el cuadro 1 muestran la variación de la estructura a lo largo del ciclo de manejo forestal. La estructura del bosque virgen (BV) es la más representativa de los bosques productivos fueguinos (Cellini et al. 1998) y las estructuras de los bosques aprovechados (BRA y BA) las esperables para el tipo de corta de reproducción propuesto. La estructura de fustal (F) refleja la capacidad de recuperación del bosque frente a un disturbio fuerte (aprovechamiento forestal mediante una tala rasa). Sin embargo, esta capacidad de recuperación puede estar influenciada por la calidad de sitio y otros factores de disturbio permanentes como el sobrepastoreo.

CUADRO 1

Estructura de los rodales muestra: bosque virgen (BV), bosque recientemente aprovechado o cosechado (BRA), bosque aprovechado o cosechado (BA) y fustal (F).

Sampled stand structure: virgin forest (BV), recently logged forest (BRA), logged forest (BA) and pole sized trees $(\mathrm{F})$.

\begin{tabular}{|l|c|c|c|c|}
\cline { 2 - 4 } \multicolumn{1}{c|}{} & B V & B R A & B A & F \\
\hline ARB (n/ha) & 411 & 90 & 53 & 1.478 \\
AB ( $\left.\mathrm{m}^{2} / \mathrm{ha}\right)$ & 59.7 & 24.2 & 12.2 & 60.7 \\
DCM (cm) & 43.0 & 58.5 & 54.1 & 22.1 \\
VTCC (m $\left.{ }^{3} / \mathrm{ha}\right)$ & 726.5 & 315.2 & II & I \\
Calidad de sitio & III & III & & 71.6 \\
\hline
\end{tabular}

$\mathrm{ARB}=$ número de árboles; $\mathrm{AB}=$ área basal; $\mathrm{DCM}=$ diámetro cuadrático medio; $\mathrm{VTCC}=$ volumen total con corteza . Clase de sitio I $\left(\right.$ IS $\left._{60}=19.8-23.2 \mathrm{~m}\right)$, clase de sitio II $\left(\right.$ IS $\left._{60}=16.5-19.8 \mathrm{~m}\right)$ y clase de sitio III (IS $\left.60=13.1-16.5 \mathrm{~m}\right)$. 
Incidencia de la cobertura en el desarrollo de los renovales del bosque virgen. $\mathrm{Al}$ analizar el efecto de la cobertura en las parcelas tomadas en el bosque virgen (BV) se detectaron diferencias significativas en el número de plantas mayores de un año (encontrándose en las menores coberturas un $200 \%$ más plantas que en las altas coberturas) (cuadro 2). La edad de las plantas también difirió en forma significativa, siendo menor el promedio de las mismas en las bajas coberturas que en las altas. Esto se debe a que las plantas se instalan con mayor facilidad en las bajas coberturas, siendo mucho menor la mortalidad que en altas coberturas. En coberturas muy cerradas sobreviven unas pocas plantas durante períodos de hasta 10 años, esperando una apertura en el dosel arbóreo que les permita desarrollarse. Por otra parte, se detectaron diferencias significativas en los residuos del suelo del bosque, encontrándose los mayores valores en altas coberturas.

Al estratificar la regeneración de más de un año, según clases de edad y altura, se encontraron diferentes distribuciones (figura 1) entre el bosque virgen (BV) y el bosque aprovechado (BA), los cuales, a su vez, poseen diferencias altamente significativas en su cobertura promedio (cuadro 3 ). Los renovales que se desarrollaron en las bajas coberturas del bosque aprovechado presentaron las mayores alturas en relación a su edad respecto del bosque virgen. En el bosque virgen la mayoría de los renovales no sobrepasaban $1055 \mathrm{~cm}$ de altura, independientemente de la edad. Estas diferencias se deben a la liberación que experimentaron los renovales al producirse la intervención forestal, lo que permitió la entrada de la luz y una mayor acumulación de agua en el suelo (Grosse 1988, Schmidt et al. 1992, Rusch 1992).

Historia forestal de los rodales e incidencia de los aprovechamientos forestales. Se encontraron diferencias significativas para el número de plántulas de más de un año, edad promedio de dichas plantas, número de plantas muertas y cobertura arbórea de los rodales estudiados según su historia forestal (cuadro 3). La lenga posee ciclos periódicos (6-8 años) de gran semillación (8-12 millones de semillas/ha) (Schmidt et al. 1995). El año 1996 fue la última semillación, incidiendo fuertemente en el número de plántulas que se encontraron en el bosque al año 1997, cuando se realizó la toma de la muestra de este trabajo. El número de plantas totales no presentó diferencias significativas (2.4 a 3.4 millones de plantas/ha) demostrando, independientemente de las coberturas de los rodales (27 a $80 \%$ ), que la lenga posee una buena dispersión y una abundante producción de semillas. Sin embargo, se encontró una mortalidad importante de plántulas debido a la competencia, íntimamente ligada a la cobertura del rodal (cuadro 3), sumamente interesante desde un punto de vista silvícola. Las plántulas se veían más saludables y robustas en el rodal con menos cobertura (BA) (hojas más grandes, mayor sistema radicular y entrenudos más largos), que las crecidas en bosques cerrados (que en su mayoría sólo presentaban un pobre desarrollo radicular y un par de hojas muy pequeñas).

\section{CUADRO 2}

Caracterización de la regeneración en dos clases de cobertura en el bosque virgen (BV). Regeneration characterisation in two crown closure classes of the virgin forest (BV).

\begin{tabular}{|l|c|c|c|c|c|}
\cline { 2 - 5 } \multicolumn{1}{c|}{} & $\begin{array}{c}\text { NP } \\
\text { (millones/ha) }\end{array}$ & $\begin{array}{c}\text { NP muertas } \\
\text { (millones/ha) }\end{array}$ & $\begin{array}{c}\text { NP }>1 \text { año } \\
(\text { millones/ha) }\end{array}$ & $\begin{array}{c}\text { E } \\
(\text { años })\end{array}$ & $\begin{array}{c}\text { Res } \\
(\%)\end{array}$ \\
\hline $\begin{array}{l}\text { Prueba de F } \\
\text { Medias para cada tratamiento }\end{array}$ & $1.563 \mathrm{~ns}$ & $1.410 \mathrm{~ns}$ & $4.504 *$ & $5.832^{*}$ & $5.144^{*}$ \\
$\begin{array}{l}\text { Cobertura (\%) } \\
50-75(\mathrm{n}=40\end{array}$ & $2.929 \mathrm{a}$ & $0.321 \mathrm{a}$ & $0.434 \mathrm{~b}$ & $1.93 \mathrm{a}$ & $24 \mathrm{a}$ \\
$75-100(\mathrm{n}=32)$ & $1.951 \mathrm{a}$ & $0.178 \mathrm{a}$ & $0.216 \mathrm{a}$ & $3.18 \mathrm{~b}$ & $41 \mathrm{~b}$ \\
\hline
\end{tabular}

$\mathrm{NP}=$ número de plantas; $\mathrm{E}=$ edad promedio de las plantas de más de un año; Res = porcentaje de residuos. Prueba de $\mathrm{F}$ : * significativo para $\mathrm{p}<0.05$; ns no significativo. Letras diferentes indican diferencias significativas para $\mathrm{p}<0.05$. 


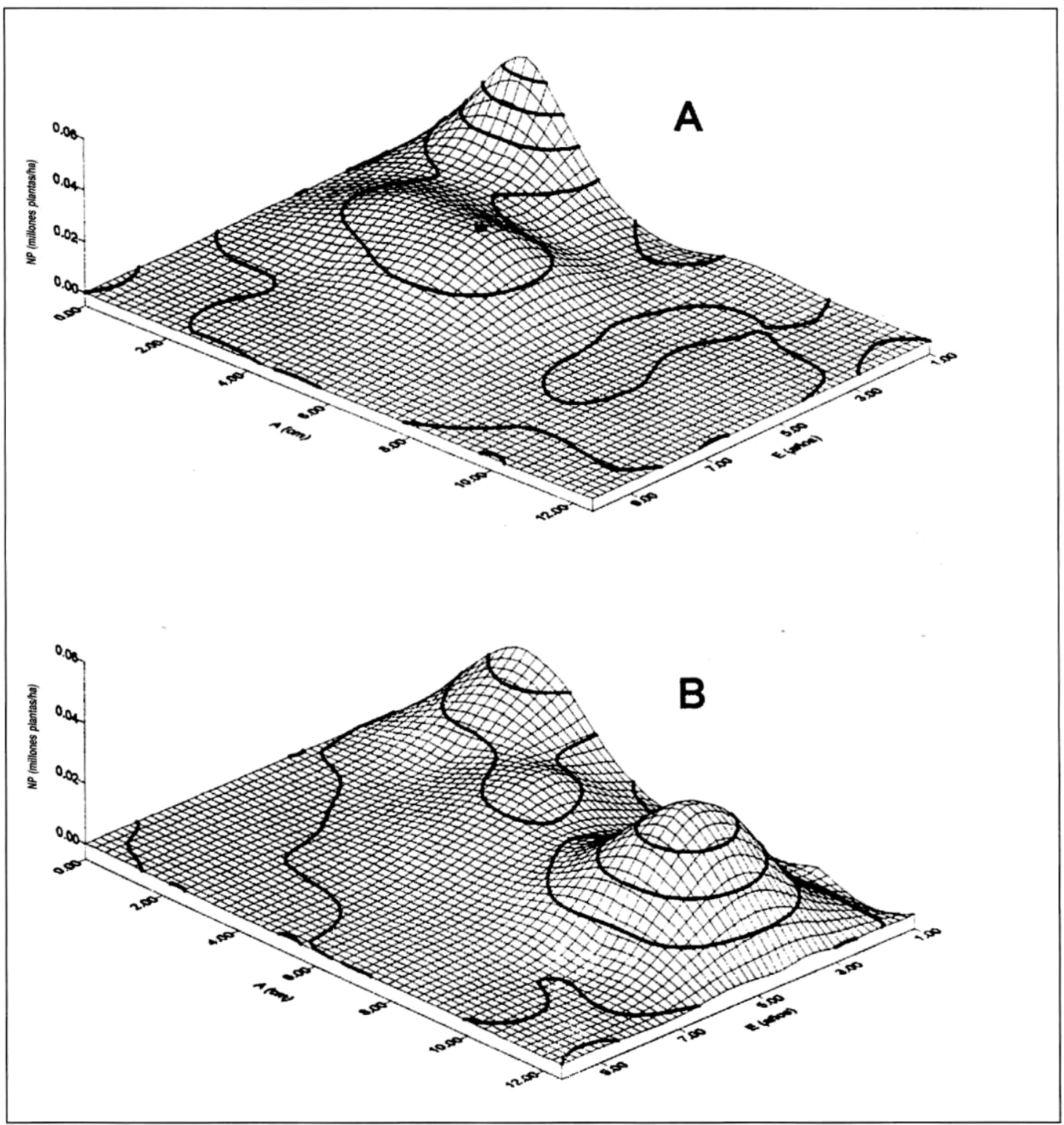

Figura 1. Número de plantas estratificadas por clases de edad (E) (años) y altura (A) (cm) para un bosque virgen (A) y un bosque aprovechado o cosechado (B).

Number of plants stratified by age (E) (years) and height $(A)(\mathrm{cm})$ for a virgin forest (A) and a logged forest (B)

Para eliminar el efecto enmascarador de la semillación del año 1996, se analizó la regeneración considerando plántulas de más de un año. El fustal (F) fue el rodal que poseía menos plántulas de más de un año, dada su alta cobertura, lo que impide el normal desarrollo de las mismas. No se encontraron diferencias entre el bosque virgen (BV) y el bosque aprovechado (BA), posiblemente debido a que en años recientes (1994-1995) casi no hubo producción de semillas (cuadro 3). Sin em- bargo, la cantidad de plántulas de más de un año existentes en el rodal es muy elevada ( 0.32 millones de plantas/ha, de aproximadamente 3 años de edad). Estos resultados permiten inferir que en un bosque virgen existe un banco permanente de plántulas que sobreviven durante 5-8 años (figura 1) esperando la apertura del dosel y que serán las primeras en desarrollar cuando se produzca el aprovechamiento del bosque. Este banco de plántulas se va recambiando a lo largo de los años (algunas 


\section{CUADRO 3}

Caracterización de la regeneración en los rodales muestreados según su historia forestal

( $\mathrm{BV}=$ bosque virgen, $\mathrm{BA}=$ bosque aprovechado, $\mathrm{F}=$ fustal $)$.

Characterisation of sampled stand regeneration according to previous management

$(\mathrm{BV}=$ virgin forest, $\mathrm{BA}=$ logged forest, $\mathrm{F}=$ pole sized trees $)$.

\begin{tabular}{|l|c|c|c|c|c|c|}
\cline { 2 - 6 } \multicolumn{1}{c|}{} & $\begin{array}{c}\text { NP } \\
\text { (millones/ha) }\end{array}$ & $\begin{array}{c}\text { NP muertas } \\
\text { (millones/ha) }\end{array}$ & $\begin{array}{c}\text { NP }>1 \text { año } \\
\text { (millones/ha) }\end{array}$ & $\begin{array}{c}\text { E } \\
\text { (años) }\end{array}$ & $\begin{array}{c}\text { Res } \\
(\%)\end{array}$ & $\begin{array}{c}\text { Cob } \\
(\%)\end{array}$ \\
\hline Prueba de F & $0.711 \mathrm{~ns}$ & $5.989 * *$ & $9.180^{* *}$ & $4.371^{*}$ & $2.408 \mathrm{~ns}$ & $231.708^{* *}$ \\
Medias para cada tratamiento & $2.440 \mathrm{a}$ & $0.250 \mathrm{~b}$ & $0.325 \mathrm{~b}$ & $2.58 \mathrm{ab}$ & $26 \mathrm{a}$ & $73 \mathrm{~b}$ \\
BV & $3.205 \mathrm{a}$ & $0.046 \mathrm{a}$ & $0.323 \mathrm{~b}$ & $3.32 \mathrm{~b}$ & $34 \mathrm{a}$ & $27 \mathrm{a}$ \\
BA & $3.390 \mathrm{a}$ & $0.299 \mathrm{~b}$ & $0.055 \mathrm{a}$ & $1.74 \mathrm{a}$ & $33 \mathrm{a}$ & $81 \mathrm{c}$ \\
F & & & & & & \\
\hline
\end{tabular}

$\mathrm{NP}=$ número de plantas; $\mathrm{E}=$ edad promedio de las plantas de más de un año; Res = porcentaje de residuos; Cob = cobertura del dosel de protección. Prueba de F: ** significativo para $\mathrm{p}<0.01$; $*$ significativo para $\mathrm{p}<0.05$; ns no significativo. Letras diferentes indican diferencias significativas para $\mathrm{p}<0.05$.

plantas mueren y otras se instalan) y es uno de los principales factores que debería de ser tenido en cuenta al realizar un plan de manejo.

Los valores de plántulas por hectárea son elevados respecto a los citados en la bibliografía: 0.191 millones de plantas/ha (Skyring - XII Región - Chile), 0.546 millones de plantas/ha (Monte Alto - XII Región - Chile) (Schmidt y Urzúa 1982), 0.34 millones de plantas/ha (Huemules Sur Chubut) (Schmaltz 1992), 0.004 a 0.167 millones de plantas/ha (Chubut) (Bava y Puig 1992), 1.933 millones de plantas/ha (XII Región - Chile) (González 1995), 0.194 millones de plantas/ha (XI Región - Chile) (Ferrando 1994), 0.031 a 1.261 millones de plantas/ha (Lago Vintter - Chubut) (Martínez-Pastur et al. 1994).

El porcentaje de residuos que impide el normal desarrollo de la regeneración no varió significativamente entre los rodales $(26 \%$ al $34 \%)$. Sin embargo, cabe destacar que el tamaño de los residuos era mucho mayor en el bosque aprovechado recientemente (BA), pero se encontraban agrupados por sectores.

Poco después de aprovechar un bosque se produce una explosión en el crecimiento de las especies del sotobosque, así como el avance sobre el bosque de varias especies que usualmente se encuentran en la vega (Fernández et al. 1998). Este aumento en el número y biomasa de especies del sotobosque se ve reflejado en el aumento de la cobertura, que presenta diferencias significativas en el bosque aprovechado (BA) respecto al bosque virgen (BV) y el fustal (F) (figura 2). Este aumento en la cobertura no afecta ni el establecimiento ni el desarrollo de la regeneración del rodal.

Las tareas de aprovechamiento (volteo, trozado y rastreo del skidder por medio de lingas) incidieron significativamente sobre la regeneración (cuadro 4). Un millón de plántulas por hectárea (41.2\%) fue destruida, siendo $0.137 \mathrm{millones} / \mathrm{ha}$ de más de un año de edad. Si bien la disminución de renovales fue significativa, el número de plántulas de más de un año instaladas (0.188 millones de plantas/ ha) es más que suficiente para asegurar la regeneración del rodal. En un bosque virgen de lenga en Monte Alto (XII Región - Chile) se encontraron 1.933 millones de plantas/ha (un rodal de $78.6 \%$ de cobertura) antes del aprovechamiento, sobreviviendo 0.674 millones de plantas/ha después de las faenas (65.1\% de pérdidas) (González 1995). En este caso las pérdidas son algo mayores, ya que las tareas de aprovechamiento fueron más intensas, pero la cantidad de renuevos sobrevivientes se consideran suficientes para una correcta regeneración del rodal. Por otra parte, Ferrando (1994) encuentra 0.194 millones de plantas/ha en un rodal antes del aprovechamiento en un bosque de Aysén (XI Región - Chile), las cuales también considera suficientes. Schmidt y Urzúa (1982) 


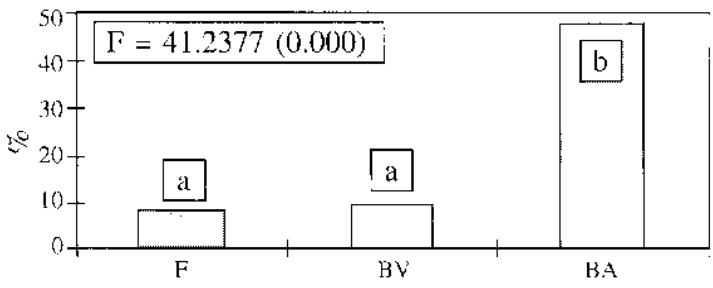

Figura 2. Porcentaje de cobertura del sotobosque en los rodales muestreados según su historia forestal $(\mathrm{BV}=$ bosque virgen, $\mathrm{BA}=$ bosque aprovechado o cosechado, $\mathrm{F}$ = fustal). Entre paréntesis se presenta la significancia para la prueba de $\mathrm{F}$ en el análisis de varianza. Letras diferentes presentan diferencias significativas para $\mathrm{p}<0.05$.

Percentage of crown clousure of the overstory in the sampled stands according to previous management $(\mathrm{BV}=$ virgin forest, $\mathrm{BA}=\operatorname{logged}$ forest, $\mathrm{F}=$ pole sized trees). Significance of $\mathrm{F}$ test in variance analysis between brackets. Different letters mean significant differences at $\mathrm{p}<0.05$.

consideran suficientes a 0.1 millones de plantas/ha con tallo lignificado para realizar una corta de protección, mientras que Schmaltz (1992) espera 0.06 millones de plantas/ha para considerar exitosa a la regeneración de un bosque de lenga.

La mayor parte del daño en la regeneración ocurre al producirse el arrastre de las trozas hasta las canchas de acopio. Sin embargo, el daño puede considerarse mínimo debido a que la remoción parcial del suelo genera condiciones propicias para la instalación de la regeneración futura en las huellas de arrastre (González 1995).

El aprovechamiento aumentó el porcentaje de residuos en forma significativa (cuadro 4) inmediatamente después de realizadas las tareas (BRA), siendo los valores algo menores a los encontrados por González (1995) (76.2\%). Gran parte de estos residuos (estrato fino) facilitarán el desarrollo de la regeneración instalada, brindando protección y mejores condiciones para el crecimiento inicial de los renuevos.

\section{CONCLUSIONES}

A partir de los altos valores de regeneración obtenidos se puede inferir que los bosques estudiados de lenga poseen una buena dispersión de semillas y una abundante y suficiente producción de plántulas (tanto si se considera o no al último máximo en la producción de semillas). Gran parte de las plántulas instaladas mueren rápidamente en altas coberturas por no tener condiciones de crecimiento propicias, sobreviviendo unas pocas. En bajas coberturas, donde se había provocado la apertura del dosel por el desmoronamiento de algún ejemplar o por el aprovechamiento del bosque, las plántulas se instalaron exitosamente, con mayor vigor y crecimiento, y con menores porcentajes de mortalidad.

\section{CUADRO 4}

Efecto del aprovechamiento sobre la regeneración del bosque virgen (BV) y el bosque recientemente aprovechado (BRA).

Harvesting effect on regeneration of the virgin forest (BV) and on recently logged forest (BRA).

\begin{tabular}{|l|c|c|c|c|c|}
\cline { 2 - 5 } \multicolumn{1}{c|}{} & $\begin{array}{c}\text { NP } \\
\text { (millones/ha) }\end{array}$ & $\begin{array}{c}\text { NP muertas } \\
\text { (millones/ha) }\end{array}$ & $\begin{array}{c}\text { NP > 1 año } \\
(\text { millones/ha })\end{array}$ & $\begin{array}{c}\text { E } \\
(\text { años })\end{array}$ & $\begin{array}{c}\text { Res } \\
(\%)\end{array}$ \\
\hline $\begin{array}{l}\text { Prueba de F } \\
\text { Medias para cada tratamiento }\end{array}$ & $4.390^{*}$ & $4.290^{*}$ & $0.185 \mathrm{~ns}$ & $6.000^{*}$ & $29.88^{* *}$ \\
BV & $2.440 \mathrm{~b}$ & $0.325 \mathrm{~b}$ & $2.58 \mathrm{a}$ & $26 \mathrm{a}$ & $73 \mathrm{~b}$ \\
BRA & $1.436 \mathrm{a}$ & $0.188 \mathrm{a}$ & $2.76 \mathrm{a}$ & $41 \mathrm{~b}$ & $57 \mathrm{a}$ \\
\hline
\end{tabular}

$\mathrm{NP}=$ número de plantas; $\mathrm{E}=$ edad promedio de las plantas de más de un año; Res = porcentaje de residuos; Cob = cobertura del dosel de protección. Prueba de F: ** significativo para $\mathrm{p}<0.01$; * significativo para $\mathrm{p}<0.05$; ns no significativo. Letras diferentes indican diferencias significativas para $\mathrm{p}<0.05$. 
Es posible encontrar un banco de plántulas en el bosque virgen que es suficiente para regenerar el bosque si se le aplica un tratamiento de corta y regeneración, debiéndose priorizar su caracterización en la toma de decisiones de un plan de manejo forestal. Sin embargo, hay que considerar que las tareas de aprovechamiento de madera dentro del bosque afectan significativamente a los renovales instalados, debiéndose tener en cuenta si se pone en peligro o no a la futura regeneración del rodal

\section{AGRADECIMIENTOS}

Al aserradero Los Castores, al Centro Austral de Investigaciones Científicas, al Instituto Nacional de Tecnología Agropecuaria y a la Universidad Nacional de la Patagonia Austral por el apoyo institucional y financiero para la realización de este trabajo. A Ricardo Vukasovic, Victoria Skrt, Paula Ferrere, Boris Díaz y Juan Manuel Cellini, quienes participaron activamente en la coordinación de los trabajos de campo.

\section{B IB LIOGR AFIA}

ARAYA VON SCHWEDLER, J.P. 1996. Rendimiento en la recuperación de un bosque floreado de Lenga (Nothofagus pumilio) a través de la corta de protección, en la XII Región. Tesis de Grado. Facultad de Ciencias Agrarias y Forestales, Universidad de Chile, 48 p.

BAVA, J., C. PUIG. 1992. Regeneración natural de lenga. Análisis de algunos factores involucrados. CIEFAP. Publicación Técnica $\mathrm{N}^{\circ} 8$, pp. 85-110.

CELLINI, J.M., C. SPAGARINO, G. MARTINEZ-PASTUR, P. PERI, R. VUKASOVIC. 1998. Rendimiento de distintos sistemas de aprovechamiento en la corta de protección de un bosque de Lenga. Actas Primer Congreso Latinoamericano de IUFRO. Valdivia (Chile), 22-28 noviembre.

FERNANDEZ, C., G. MARTINEZ-PASTUR, P. PERI, R. VUKASOVIC. 1997. Thinning schedules for Nothofagus pumilio forest in Patagonia, Argentina. Actas del XI Congreso Forestal Mundial. Volumen 3: D. Función productiva de los bosques. Antalya, 13-22 octubre.

FERNANDEZ, C, G. STAFFIERI, G. MARTINEZ-PASTUR, P. PERI. 1998. Cambios en la biodiversidad del sotobosque a lo largo del ciclo del manejo forestal de la Lenga. Actas
Primer Congreso Latinoamericano de IUFRO. Valdivia (Chile), 22-28 noviembre.

FERRANDO T., M. 1994. Estructura de un bosque de lenga de la XI Región, y rendimiento volumétrico en la corta de protección. Tesis de Grado. Facultad de Ciencias Agrarias y Forestales, Universidad de Chile, $57 \mathrm{p}$.

GONZALEZ R., S.A. 1995. Rendimiento volumétrico en la corta de protección de un bosque de lenga en Monte Alto, XII Región. Tesis de Grado. Facultad de Ciencias Agrarias y Forestales, Universidad de Chile, $51 \mathrm{p}$.

GROSSE, H. 1988. "Desarrollo de plantas de los géneros Fagus y Nothofagus en función de la luminosidad. Revisión bibliográfica", Ciencia e Investigación Forestal 2(3): 114120.

LEMMON, P. 1957. "A new instrument for measuring forest overstory density", Journal of Forestry 55(9): 667-668.

MARTINEZ-PASTUR, G., C. FERNANDEZ, P. PERI. 1994. "Variación de parámetros estructurales y de composición del sotobosque para bosques de Nothofagus pumilio en relación a gradientes ambientales indirectos", Ciencias Forestales 9 (1-2): 11-22.

MARTINEZ-PASTUR, G., P. PERI, R. VUKASOVIC, S. VACCARO, V. PIRIZ CARRILlO. 1997. "Site index equation for Nothofagus pumilio forests", Phyton 6(1/2): 55-60.

PERI, P., G. MARTINEZ-PASTUR, B. DIAZ, F. FUCARACCIO. 1997. Uso del índice de Sitio para la construcción de ecuaciones estándar de volumen total de fuste para lenga (Nothofagus pumilio) en Patagonia Austral. Actas II $^{\circ}$ Congreso Forestal Argentino y Latinoamericano. Tomo Bosques Nativos y Protección Ambiental, p. 309316. Posadas, 13-15 agosto.

REBERTUS, A., T. VEBLEN. 1993. "Structure and tree-fall gap dynamics of old-growth Nothofagus forests in Tierra del Fuego, Argentina", Journal of Vegetation Science 4: 641-654.

RECHENE, C., H. GONDA. 1992. Propuesta metodológica y plan de manejo preliminar para lenga en sitios de calidad media de Chubut. Actas del seminario de manejo forestal de la lenga y aspectos ecológicos relacionados. CIEFAP. Publicación Técnica $\mathrm{N}^{\circ}$ 8, pp 126-159.

RUSCH, V. 1992. Principales limitantes para la regeneración de la lenga en la zona NE de su área de distribución: variables ambientales en claros del bosque. CIEFAP. Publicación Técnica $\mathrm{N}^{\circ} 8$, pp. 61-73.

SCHMALTZ, J. 1992. Aspectos de regeneración de Haya y Lenga - una comparación. CIEFAP. Publicación Técnica $\mathrm{N}^{\circ} 8$, pp. 74-84.

SCHMIDT, H., A. URZUA. 1982. Transformación y manejo de los bosques de Lenga en Magallanes. Universidad de Chile. Ciencias Agrícolas 11. 62 p.

SCHMIDT, H., J. CALDENTEY, T.P. GAERTIG. 1992. Informe Lenga 1992: Análisis silvicultural de los ensayos. Universidad de Chile - CONAF, 37 p.

SCHMIDT, H., J. CALDENTEY, S. DONOSO. 1995. Informe 1995 Investigación sobre el manejo de la Lenga - XII Región. Universidad de Chile - CONAF, 40 p.

VATASAN, G. 1983. "Notes on the regeneration of beech after logging on Mt Giluwe", Klinkii 2(3): 65-68. 